NIMA DADASHZADEH, Ph.D. ${ }^{1,2}$

(Corresponding Author)

E-mail: dadashzadeh@itu.edu.tr

MURAT ERGUN, Ph.D. ${ }^{3}$

E-mail: ergunmur@itu.edu.tr

SERCAN KESTEN, Ph.D. ${ }^{4}$

E-mail: sercan.kesten@isikun.edu.tr

MARIJAN ŽURA, Ph.D. ${ }^{2}$

(Corresponding Author)

E-mail: marijan.zura@fgg.uni-lj.si

${ }^{1}$ ITÜ Graduate School of Science,

Engineering and Technology

Ayazaga Kampüsü, 34469 Maslak/Istanbul, Turkey

2 Traffic Technical Institute, Faculty of Civil and Geodetic

Engineering, University of Ljubljana

Jamova cesta 2, 1000 Ljubljana, Slovenia

${ }^{3}$ ITÜ Civil Engineering Faculty, Transportation Eng. Division

Ayazağa Kampüsü, 34469 Maslak/Istanbul, Turkey

${ }^{4}$ Işık University, Civil Engineering Division

Maslak Kampüsü, 34398 Maslak/Istanbul, Turkey
Swarm Intelligence in Transportation Engineering

Original Scientific Paper

Submitted: 22 Oct. 2018

Accepted: 12 June 2019

\title{
AN AUTOMATIC CALIBRATION PROCEDURE OF DRIVING BEHAVIOUR PARAMETERS IN THE PRESENCE OF HIGH BUS VOLUME
}

\begin{abstract}
Most of the microscopic traffic simulation programs used today incorporate car-following and lane-change models to simulate driving behaviour across a given area. The main goal of this study has been to develop an automatic calibration process for the parameters of driving behaviour models using metaheuristic algorithms. Genetic Algorithm (GA), Particle Swarm Optimization (PSO), and a combination of GA and PSO (i.e. hybrid GAPSO and hybrid PSOGA) were used during the optimization stage. In order to verify our proposed methodology, a suitable study area with high bus volume on-ramp from the 0-1 Highway in Istanbul has been modelled in VISSIM. Traffic data have been gathered through detectors. The calibration procedure has been coded using MATLAB and implemented via the VISSIM-MATLAB COM interface. Using the proposed methodology, the results of the calibrated model showed that hybrid GAPSO and hybrid PSOGA techniques outperformed the GA-only and PSO-only techniques during the calibration process. Thus, both are recommended for use in the calibration of microsimulation traffic models, rather than GA-only and PSO-only techniques.
\end{abstract}

\section{KEY WORDS}

traffic simulation models; calibration; driving behaviour; Genetic Algorithm; Particle Swarm Optimization; VISSIM;

\section{INTRODUCTION}

In recent years, advances in computing hardware technology and new traffic engineering applications have led to greater use of traffic simulations in the analysis of complex interactions between various traffic components [1]. Microscopic traffic models are those based on the principle of the movement of each individual vehicle or pedestrian included in the traffic, taking account of actions and decisions such as acceleration, deceleration, and lane/trajectory changes in response to the surrounding conditions [2]. A wide variety of traffic simulation software is either commercially or freely available on the market. Some examples of this microsimulation software include VISSIM, AIMSUN, CORSIM, PARAMICS, MITSimLab, FRESIM, DRACULA, and SUMO. Although a wealth of microscopic traffic simulation software is available, traffic simulation studies still lack a unified perspective in terms of mimicking the real-world conditions. Having a finetuned and best-matched simulation model which represents the real-life behaviour of drivers, is of pivotal importance to traffic engineers. Thus, before any analysis can take place, models need to be calibrated to be able to represent real-life conditions. The calibration process has the objective of finding the statistically significant values of model parameters based on data collected from the field [3]. From these samples, the performance of a traffic model can be determined by employing statistical analysis with respect to various measures [4, 5].

The validity of the model can be done simply in terms of the probability that the difference between the observed and simulated output is of less than a 
delineated tolerable difference [2]. Various optimization methods have been employed to minimize the difference between the observed and simulated outputs. These include GA [6-8], SPSA [9], PSO [10], OQMS [4], and a combination/comparison of various of these [5, 11]. The aforementioned studies show that GA is the optimization method most favoured by researchers because of its ease-of-implementation. However, no information exchange is taking place between individuals during the GA process. For instance, in the selection stage, the members of the initial population have no direct competition to being selected and neither do parents in the crossover stage experience any information exchange with each other or that of the offspring created by them. On the other hand, when a mutation occurs, this mutant lacks the right direction. These are the reasons for lower performance of GA compared to other techniques.

This study outlines an automatic calibration process for driving behaviour model parameters using metaheuristic algorithms. The Genetic Algorithm (GA), Particle Swarm Optimization (PSO), and a combination of GA and PSO (i.e. hybrid GA and hybrid PSO) were utilized for optimization purposes. A case study of an urban highway section is presented within the microsimulation environment of VISSIM [12]. The main contribution provided by the proposed methodology is a hybrid method for overcoming the limitation of single optimization algorithms in order to yield better results in a fully automatic way. Four optimization algorithms - namely, GA, PSO, GAPSO, and PSOGA - were coded in MATLAB and the results compared in order to find the most suitable to be used in the VISSIM calibration process. The Component Object Model (COM) ability of VISSIM was employed to provide a bridge for the exchange of information between MATLAB and VISSIM and an automated calibration process. Although hybrid PSO and hybrid GA are used in other fields of study [13-16], this study is the first that implemented a combination for the calibration of traffic microsimulation parameters.

Other sections of the study deal with a brief literature review of the driving behaviour models, parameters, and optimization algorithms. The proposed calibration methodology is described in Section 3. This is followed by a real-world application of the proposed calibration methodology as tested on an urban highway in Istanbul, Turkey. Section 5 discusses the results of the calibration and optimization process in detail, followed by our conclusion and suggestions for future research.

\section{BACKGROUND}

In this section, VISSIM driver behaviour models including car-following, lane-change, their parameters' description, and optimization methods are discussed briefly.

\subsection{VISSIM Car-following and Lane-change models description}

Many studies opt to use default car-following and lane-changing parameters. However, the traffic composition, network geometry, vehicle ages, engine size, and (most of all) driver behaviour vary significantly in different parts of the world. Thus, the default parameters of the simulation software should be carefully examined in order to obtain reliable results. As an example, it has been noted that lane-changing is a highly strong characteristic of Istanbul traffic and drivers are frequent and aggressive in cutting and overtaking, taking every opportunity to change lanes at the slightest opening [17]. As explained in [12], the two models of driving behaviour parameters are Wiedemann 74 (W74) and Wiedemann 99 (W99). The W74 model, generally, has been used for urban arterials and merging areas. The W99 has been utilized in modelling freeways and diverging areas. Tables 1-4 outline the general parameters, lane-changing, W74, and W99 models parameters respectively. The first column contains the ID of each parameter used by VISSIM during COM interface, along with the parameter description, their range, and default values in other columns.

\subsection{Evolutionary Algorithms}

Generally, all EAs consist of a number of common steps, including initialization, variables/parameters definition, objective function definition, iteration steps, stopping criteria. There is a small difference in the procedure required for various types of EA. The two most frequently used EA algorithms are GA and PSO.

Table 1 - General Parameters

\begin{tabular}{||l|l|c|c||}
\hline \multicolumn{1}{|c|}{ IDrivingBehavior } & \multicolumn{1}{|c|}{ Parameter description } & Range & Default \\
\hline \hline LookBackDistMax & Max. look back distance $[\mathrm{m}]$ & $50 \sim 200$ & 150 \\
\hline LookAheadDistMax & Max. look ahead distance $[\mathrm{m}]$ & $100 \sim 300$ & 250 \\
\hline ObsrvdVehs & No. of observed preceding vehicles [veh] & $1.00 \sim 5.00$ & 2.00 \\
\hline StandDist & Standstill distance in front of static obstacles [m] & $0.00 \sim 3.00$ & 0.50 \\
\hline
\end{tabular}


Table 2 - Lane-changing model parameters

\begin{tabular}{||l|l|c|c||}
\hline \multicolumn{1}{|c|}{ IDrivingBehavior } & \multicolumn{1}{|c|}{ Parameter description } & Range & Default \\
\hline \hline DecelRedDistOwn & Reduction rate for leading (own) vehicle $[\mathrm{m}]$ & $100 \sim 200$ & 200 \\
\hline AccDecelOwn & Accepted deceleration for leading (own) vehicle $\left[\mathrm{m} / \mathrm{s}^{2}\right]$ & $-3.00 \sim 0.50$ & -1.00 \\
\hline MinHdwy & Min. spacing (headway) [m] & $0.50 \sim 3.50$ & 0.50 \\
\hline SafDistFactLnChg & Safety distance reduction factor & $0.10 \sim 0.60$ & 0.60 \\
\hline CoopDecel & Max. deceleration for cooperative lane-change/braking [m/s $\left.{ }^{2}\right]$ & $-6.00 \sim 3.00$ & -3.00 \\
\hline CoopLnChgSpeedDiff & $\begin{array}{l}\text { Max. speed difference for cooperative lane-change/braking } \\
{[\mathrm{m} / \mathrm{s}]}\end{array}$ & $5.00 \sim 20.00$ & 10.80 \\
\hline MaxDecelOwn & Max. deceleration for leading (own) vehicle $\left[\mathrm{m} / \mathrm{s}^{2}\right]$ & N.A & -4.00 \\
\hline MaxDecelTrail & Max. deceleration for following (trailing) vehicle $\left[\mathrm{m} / \mathrm{s}^{2}\right]$ & N.A & -3.00 \\
\hline DecelRedDistTrail & Reduction rate for following (trailing) vehicle $[\mathrm{m}]$ & N.A & 200 \\
\hline AccDecelTrail & Accepted deceleration for following (trailing) vehicle $\left[\mathrm{m} / \mathrm{s}^{2}\right]$ & N.A & -0.50 \\
\hline
\end{tabular}

Table 3 - Wiedemann 74 car-following model parameters

\begin{tabular}{||l|l|c|c||}
\hline \multicolumn{1}{|c|}{ IDrivingBehavior } & \multicolumn{1}{|c|}{ Parameter description } & Range & Default \\
\hline \hline W74ax & Average standstill distance & $0.50 \sim 2.50$ & 2.00 \\
\hline W74bxAdd & Additive factor for security distance & $0.70 \sim 4.70$ & 2.00 \\
\hline W74bxMult & Multiplicative factor for security distance & $1.00 \sim 8.00$ & 3.00 \\
\hline
\end{tabular}

Table 4 - Wiedemann 99 car-following model parameters

\begin{tabular}{|c|c|c|c|}
\hline IDrivingBehavior & Parameter description & Range & Default \\
\hline W99cC0 & Desired distance between lead and following vehicle [m] & $0.60 \sim 3.05$ & 1.50 \\
\hline W99CC1DISTR & $\begin{array}{l}\text { Headway Time }[\mathrm{s}] \\
\text { Desired time between lead and following vehicle }\end{array}$ & $0.50 \sim 1.50$ & 0.90 \\
\hline W99CC2 & $\begin{array}{l}\text { Following Variation }[\mathrm{m}] \\
\text { Additional distance over safety distance that a vehicle requires }\end{array}$ & $1.52 \sim 6.10$ & 4.00 \\
\hline W99cC3 & $\begin{array}{l}\text { Threshold for Entering ‘Following' State [s] } \\
\text { Time in seconds before a vehicle starts to decelerate to reach safety } \\
\text { distance (negative) }\end{array}$ & $-15.00 \sim-4.00$ & -8.00 \\
\hline W99cC4 & $\begin{array}{l}\text { Negative "Following Threshold" [m/s] } \\
\text { Specifies variation in speed between lead and following vehicle }\end{array}$ & $-0.61 \sim 0.03$ & -0.35 \\
\hline W99CC5 & $\begin{array}{l}\text { Positive "Following Threshold" [m/s] } \\
\text { Specifies variation in speed between lead and following vehicle }\end{array}$ & $0.03 \sim 0.61$ & 0.35 \\
\hline W99cc6 & Speed dependency of oscillation $[1 / \mathrm{ms}]$ & $7.00 \sim 15.00$ & 11.44 \\
\hline W99CC7 & $\begin{array}{l}\text { Oscillation Acceleration: Acceleration during the oscillation process } \\
{\left[\mathrm{m} / \mathrm{s}^{2}\right]}\end{array}$ & $0.15 \sim 0.46$ & 0.25 \\
\hline W99cc8 & Standstill Acceleration $\left[\mathrm{m} / \mathrm{s}^{2}\right]$ & $2.50 \sim 5.00$ & 3.50 \\
\hline W99cc9 & Acceleration with $80 \mathrm{~km} / \mathrm{h}\left[\mathrm{m} / \mathrm{s}^{2}\right]$ & $0.50 \sim 2.50$ & 1.50 \\
\hline
\end{tabular}

Evolutionary algorithms differ from a classical, derivative-based, optimization algorithm in two main ways, as summarized in Table 5.

\section{Genetic Algorithm}

GA [18] is one of the best-known population-based (biological) example among EAs. It has been used for both binary and continuous forms in single and multi-objective optimization processes. All GA forms generally possess common rules including selection, crossover, and mutation, at each step creating new chromosomes (generation) from the existing ones. At each stage, GA selects initial population (generation) randomly, selects parents from among the current population, and combines selected parents to produce offspring (children) for the next generation during the crossover process using various methods such as single-, double-point crossover, or uniform crossover. There are several tuning elements which are involved in GA, including the number of initial population, maximum iteration number, crossover percentage, mutation percentage, mutation rate, etc. Detailed information concerning how sensitivity analysis of tuning elements influences the GA is described in Section 5 
Table 5 - Classical and evolutionary algorithm differences

\begin{tabular}{|l|l||}
\hline \multicolumn{1}{|c|}{ Classical algorithm } & \multicolumn{1}{|c|}{ Evolutionary algorithm } \\
\hline \hline $\begin{array}{l}\text { Generates a single point at each iteration. The sequence } \\
\text { of points approaches an optimal solution. }\end{array}$ & $\begin{array}{l}\text { Generates a population of points at each iteration. The best } \\
\text { point in the population approaches an optimal solution. }\end{array}$ \\
\hline $\begin{array}{l}\text { Selects the next point in the sequence by a deterministic } \\
\text { computation. }\end{array}$ & $\begin{array}{l}\text { Selects the next population by computation which uses } \\
\text { random number generators. }\end{array}$ \\
\hline \hline
\end{tabular}

of [19]. We use uniform random selection for initial population, an arithmetic crossover (a kind of uniform crossover), create and add noise (random number) using Normal Distribution (with mean $=0$ and variance=sigma) for improving selected offspring during the mutation stage, and following settings for the GA elements given in Table 6.

\section{Particle Swarm Optimization}

PSO, firstly introduced by [20], is also a population-based algorithm but it uses particle swarms of intelligence ability; for instance, the behaviour of fish when they are confronted with a shark. PSO is an algorithm for continuous variables, but with some modification it can be used in discontinuous optimization problems, too [21]. PSO begins with a determination of the position and velocity of each individual (particle) and proceeds with a calculation of the objective function based on that particle's location. Then, the objective function values are to be compared with global objective function values, with the better one assigned as a global objective value. The new velocity and position of the particles are calculated based on the best particle information. The main advantage of PSO is that an information flow exists between all particles at each moment. This means that all particles use other information to find the best solutions. This capacity of PSO is used for solving GA limitation issues, particularly during the selection, crossover, and mutation stages.
There are several stopping criteria for both GA and PSO, including Max Stall Iterations, Function Tolerance, Max Iterations, OutputFcn or PlotFcn, Objective Limit, and Max Stall Time. However, we use only Max Number of Iterations (Maxlt) as stopping criteria for the proposed method because we wanted to let all the methods perform a similar number of function evaluations.

\section{PROPOSED CALIBRATION METHODOLOGY}

There exist two types of methods for Driving Behaviour Parameters (DBP) calibration; (1) calibration of DBP using trajectory data (lane-changing, acceleration, deceleration, etc.) extracted from video files using image-processing techniques [22-24]; (2) calibration of DBP using traffic flow measurement data (volume, speed, etc.) collected by detectors [6, 25, 26]. As we do not possess the capabilities for automatic image processing, we used the latter approach. Figure 1 illustrates the whole picture of the proposed methodology. Each part of the flowchart is described in detail in the following sections. As noted in Section 2.2, all EAs consist of a number of common steps (top of the optimization flow) while each algorithm has its own operator (bottom of the optimization flow).

\subsection{Objective (i.e. error) function definition}

As noted in the background, there are many single and multi-objective functions used to minimize

Table 6 - GA operator settings for the current proposed methodology

\begin{tabular}{|l|l|}
\hline MaxIt=40; & Maximum Number of Generation (stopping criteria) \\
nPop=10; & Population Size \\
pCrossover=0.8; & Crossover Percentage \\
nCrossover=round (pCrossover*nPop/2)*2; & Number of Parents (Offspring) \\
pMutation=0.3; & Mutation Percentage \\
nMutation=round (pMutation*nPop); & Number of Mutants \\
Mu=0.1; & Mutation rate \\
\hline
\end{tabular}

Table 7 - PSO operator settings for the current proposed methodology

\begin{tabular}{|c|c|}
\hline $\begin{array}{l}\text { MaxIt=40; } \\
\text { nPop=10; } \\
\text { phil=2.05; } \\
\text { phi2=2.05; } \\
\text { phi=phi1+phi2; } \\
\text { chi=2/(phi-2+sqrt (phi^2-4*phi)); } \\
\text { W=chi; } \\
\text { c1=chi }{ }^{\star} \text { phi1; } \\
\text { c2=chi }{ }^{\star} \text { phi2 }\end{array}$ & $\begin{array}{l}\text { Maximum Number of Iterations } \\
\text { Swarm (Population) Size } \\
\text { Definition of Constriction Coefficients } \\
\text { Inertia Weight } \\
\text { Personal Learning Coefficient } \\
\text { Global Learning Coefficient }\end{array}$ \\
\hline
\end{tabular}




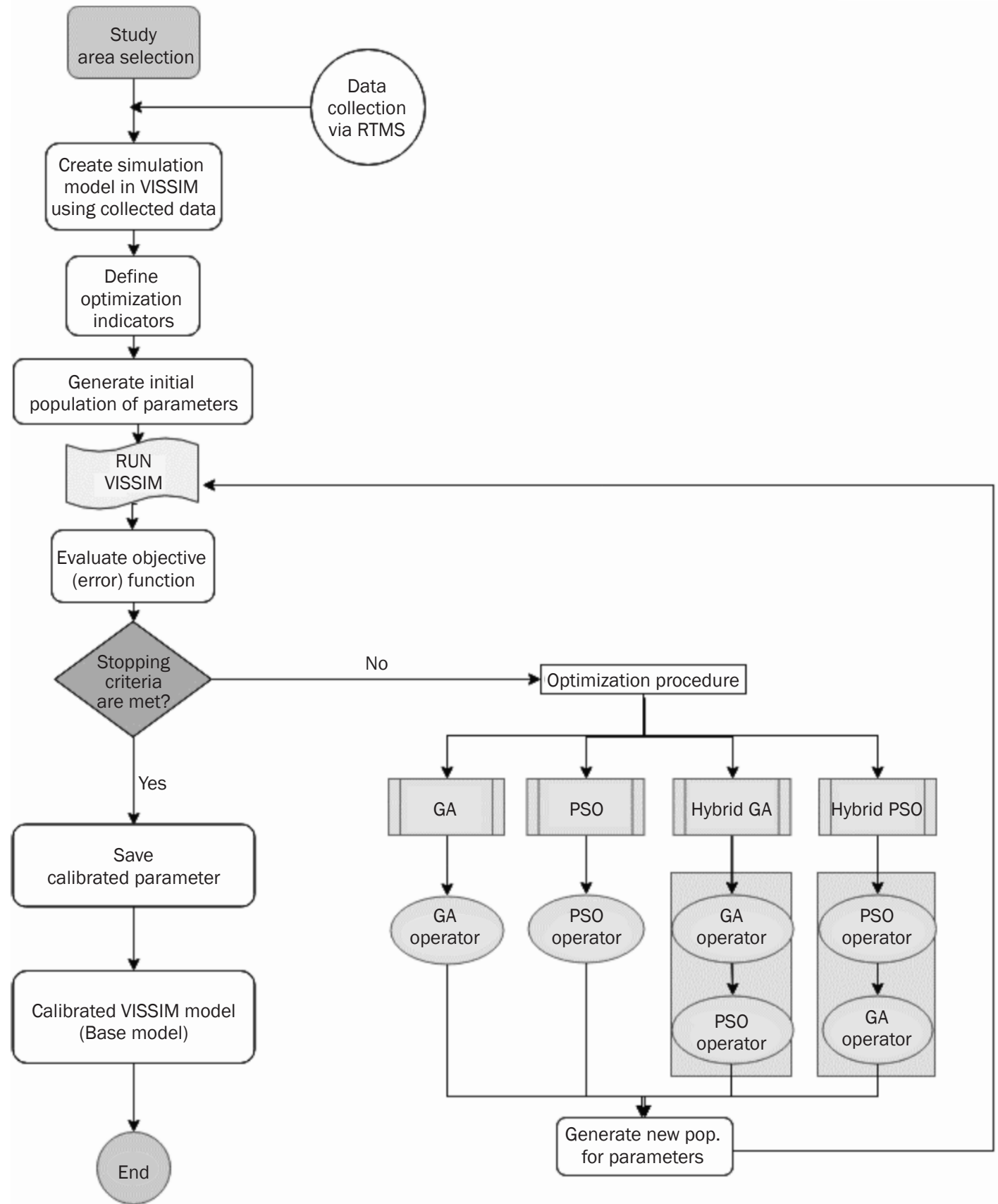

Figure 1 - Flowchart of the proposed methodology

the error of simulated and observed data. The Root Mean Square Error (RMSE) [5, 27, 28] and the Mean Absolute Normalized Error (MANE) [4, 11, 29, 30] fall among several multi-objective functions used in previous studies for the calibration of VISSIM simulation model parameters and are widely used around the world. The developed code, here, can perform the optimization process based on both single (e.g. speed-only, volume-only, and occupancy rate) and multi-objective functions. In this study, we tried to minimize the error between simulated and observed data utilizing MANE and RMSE objective functions formula:
Minimize $Z(M A N E)$

$=\frac{1}{N} \sum_{j=1}^{N}\left(\frac{\left|V_{o b s_{j}}-V_{\text {sim }}\right|}{V_{o b s_{j}}}+\frac{\left|S_{\text {obs } j}-S_{\text {sim }}\right|}{S_{o b s j}}\right)$

Minimize Z(RMSE)

$=\sqrt{\frac{1}{N} \sum_{j=1}^{N}\left(S_{o b_{j}}-S_{\text {sim }}\right)^{2}}$

w.r.t the constraints: $L b_{X_{i}} \leq X_{i} \leq U b_{X_{i}}$

where:

Z $\quad$-general form of objective function (here based on speed and traffic volume); 
$X_{i} \quad$-vector of continues parameters (e.g. W74 or W99 Car-following models

+ Lane-change model parameters);

$L b_{X_{i}}, U b_{X_{i}}$-lower and upper value of parameter $X_{i}$ (e.g. $C C 1: L b_{c c 1}=0.5$ and $U b_{c c 1}=1.5 \mathrm{~s}$ );

$V_{o b s j}, S_{o b s j}$-observed traffic volume and speed collected by detectors;

$V_{\text {simj }}, S_{\text {simj }}$-simulated traffic volume and speed by VISSIM;

$N \quad$-total number of data collection intervals (e.g. for one hour observation $(3,600 \mathrm{sec})$ with two minutes intervals (120 sec), it is equal: $N=3,600 / 120=30$ ).

\subsection{Programming of the optimization process}

Implementation of the iteration step of the proposed flowchart in Figure 1 needs to be programmed using the aforementioned required information on GA and PSO algorithms. MATLAB was used to develop a code for the fully-automatic calibration procedure. To this end, the COM interface features between VISSIM and MATLAB are studied to integrate the code written in MATLAB with microscopic model simulated in VISSIM. For the optimization process, during the calibration procedure, one of GA, PSO, or a combination of both can be used. We coded our own algorithm structures for GA, PSO, and hybrid without utilizing the optimization toolbox of MATLAB. This increased the flexibility of our proposed methodology and gave us the opportunity to extend/improve the code for further studies noted in the conclusion part. Possible options for the optimization process in the proposed methodology were as follows: GA-only, PSO-only, PSOGA (called hybrid PSO), GAPSO (called hybrid GA).

There are several possible combinations of GA and PSO for the hybrid method used in other fields of studies [14, 31, 32]. All try to use the advantages of local search capability of GA and social thinking ability of PSO as both algorithms have strengths and weaknesses. They concluded that the combination of standard PSO and standard GA resulted in better performance compared to the use of single algorithms. Some of them used only one or more operators of GA such as using crossover and mutation operators in the PSO for improving and balancing PSO's exploration and exploitation ability [14]. Others use the ability of PSO in saving and updating the personal and global best in GA [33]. In the proposed methodology, as seen in the optimization process flow of Figure 1, the initial population of PSO is created and assigned by the GA operator. The total numbers of iterations are equally shared by GA and PSO, if MaxSubltGA and MaxSubltPSO are set to 1 . In other words, in every iteration, the code runs one GA and one PSO operator. A user can also modify the share of using the GA and PSO operators in every iteration by increasing or decreasing the number of MaxSubltGA and MaxSubltPSO. The order of the operation of the GA operator and the PSO operator is related to the hybrid type used for the calibration. As seen below for instance, in hybrid GA-PSO, initial position and velocity of particles (here driving behaviour parameters) are determined randomly over the search space. Then, the crossover and mutation operators of GA are applied for each particle in swarm separately to improve the diversity of the population and find better sets of parameters. The information (position, velocity) of each particle was calculated and compared with their previous information and also with the global best. If new information is better than the previous personal best and global bests both of them were updated based on new information. The solutions obtained by the GA operator are given as initial population of the PSO; PSO operator starts to search within the search space around the best particle by introducing swarm intelligence as explained in Section 2.2. It attracts the particles toward the actual best position while maintaining the parameters diversity to gain the new best in every iteration compared to the previous iteration. The proposed Pseudo code of GAPSO algorithm for VISSIM calibration is presented below.

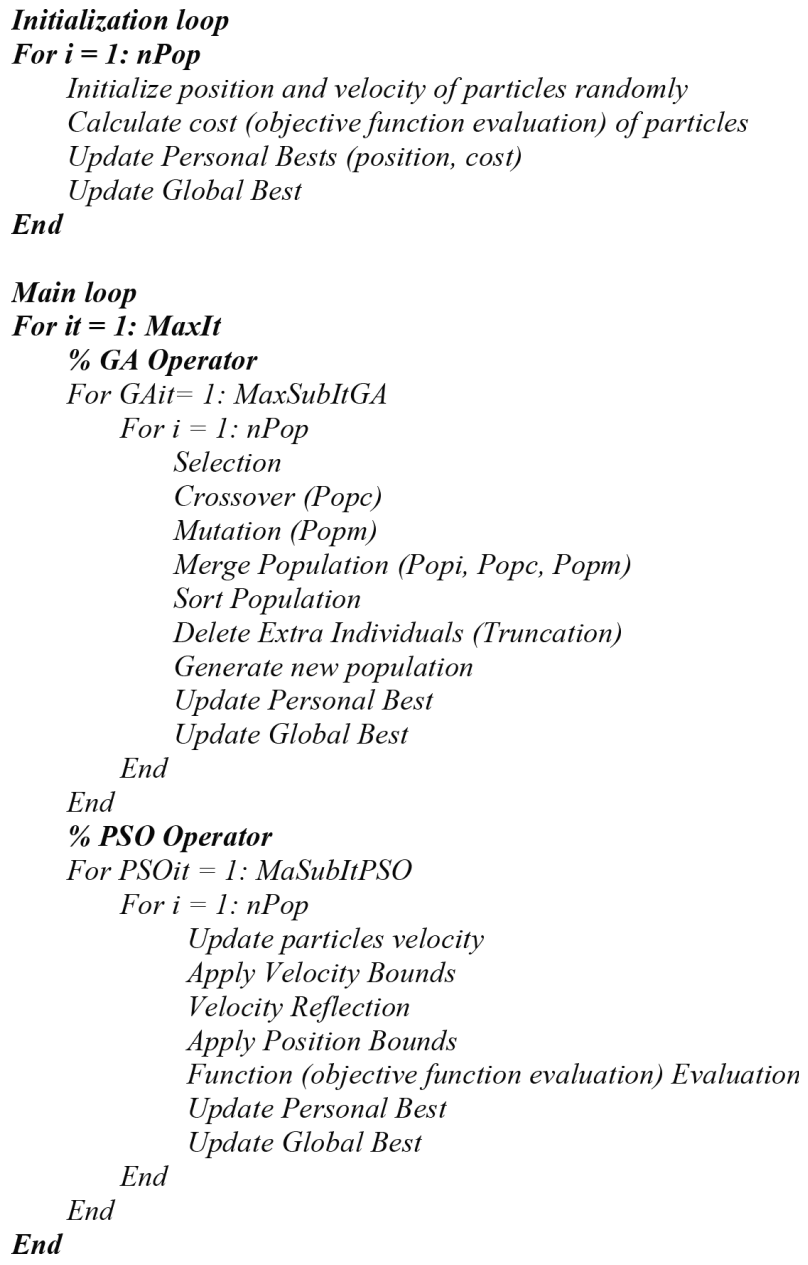




\section{REAL-WORLD APPLICATION}

In order to test the proposed calibration method, a case study area was selected and the proposed methodology implemented on the simulated highway stretch. For this purpose, one segment of the 0-1 Highway in Istanbul, Turkey, specifically the Yıldız junction, was selected. A bottleneck area is formed at Yıldız junction through one mixed traffic lane and a spatial bus priority lane merging into three-lane mainline [34]. The driving and lane-changing behaviour in this specific section are observed to be peculiar due to its distinct geometry and traffic composition, as shown in Figure 2.

\section{Data collection procedure}

As shown in Figure 2, the Yıldız merging area of the 0-1 highway consists of three lanes with mixed traffic flow per direction. Due to the distribution of residential and business districts in Istanbul, the majority of Bosporus crossings go from the Asian side to the European side in the morning hours, with the opposite flow appearing in the evening hours $[35,36]$. This study just considered the flows in the European to Asianside direction. There are two Remote Traffic Microwave Sensor (RTMS) devices installed in the upstream (No. 303) and downstream (No. 60) of the bottleneck area which provide presence indication and accurate measurements of volume, occupancy, and speed with a detection range (increment) of $0.4 \mathrm{~m}(1.3 \mathrm{ft})$ for each two-minute time interval in $7 / 24$ duration. The one-week data (07.05.18 - 14.05.18) of RTMS detectors from 6:00 AM to 10:00 PM provided by Istanbul Metropolitan Municipality, Traffic Control Center (IMMTCC) was analysed in order to select the start and end time-point of the capacity drop phenomena during the morning and evening peak hours. In this study, traffic conditions between 14:30-15:30 are modelled including an un-congested flow, transition condition, and congested flow conditions.

\section{Simulation of the study area using VISSIM}

The well-known microsimulation software, VISSIM version 10 [12] was used to create a microscopic model of the Yıldız merging area. In the base model, we use the default values of parameters for driving behaviour

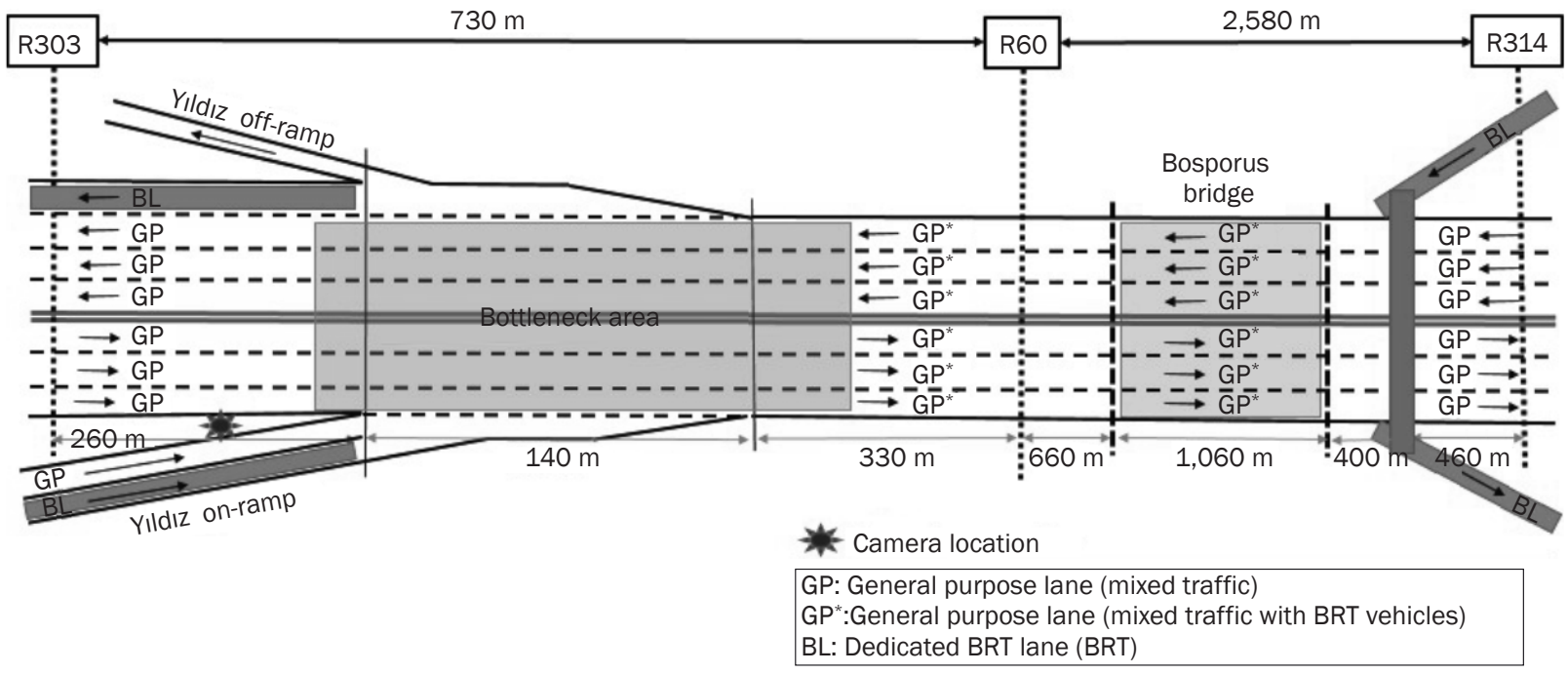

R303: RTMS detector No. 303 (mixed traffic excl. BRT vehicle) R60: RTMS detector No. 60 (mixed traffic incl. BRT vehicle) R314: RTMS detector No. 314 (mixed traffic excl. BRT vehicle)

Figure 2 - A schematic view of Yıldız merging area in 0-1 Highway in Istanbul
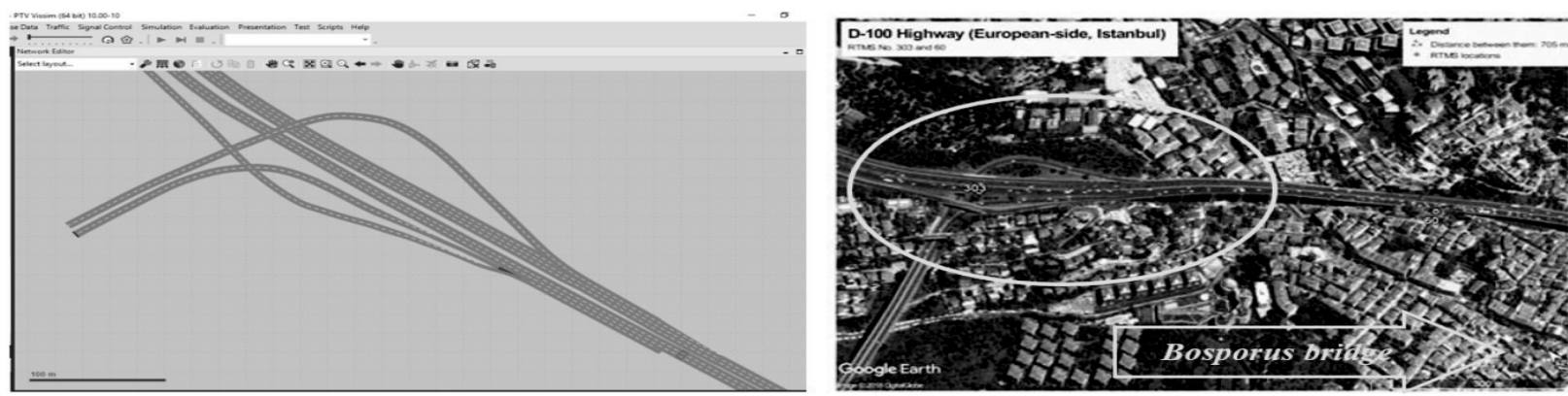

Figure 3 - Modelled study area by VISSIM (left) vs. Bird's eye view of study area (right); Source: Google Earth 
models. After simulation, we compared simulated traffic volumes and speeds at detectors for every two-minute interval with the measured values. A comparison between the modelled data and observed data reveals that there is a significant difference between these two sets of data. It justifies the need for formulating a calibrated model based on actual traffic condition before making a scenario analysis. The results of the optimization process and the driving behaviour model parameters using the proposed calibration method are discussed in detail in Section 5.

\section{VISSIM simulation and evaluation settings}

The following values for the simulation and evaluation attributes were set. As noted below, the total simulation time (period time) was calculated as 300 $+3,600=3,900 \mathrm{sec}$. We assumed 300 seconds as a warm-up time at the beginning of the simulation period. Data collection was done in just 60-minute simulation period with a two-minute time interval (120 sec) excluding warm-up periods. In order to decrease the simulation time as well, 'QuickMode' and 'UseMaxSimSpeed' attributes were activated. In order to eliminate the stochastic discrepancy, in each scenario, five independent runs with the same initial condition and different speeds were made and an average of the total time was recorded. To this end, the simulation settings used in VISSIM were as follows: initial random speed $=40$, speed increment $=3$, number of runs $=5$, step time $($ resolution $)=5$, simulation time $=3,900$ with $\max$ speed for Simulation ('UseMaxSimSpeed', true and 'QuickMode', 1).

\section{RESULTS AND DISCUSSION}

In our study, eleven parameters to be optimized using the proposed methodology were selected. They were selected from the general parameters ("LookBackDistMax"," LookAheadDistMax", "StandDist”,
"ObsrvdVehs"), W74, and lane-change model parameters. Table 8 presents the obtained MANE and RMSE values. As shown in Table 8, the simulation with default values of the driving behaviour and lane-change parameters yielded worse MANE and RMSE values compared to simulations with calibrated parameters using any of the metaheuristic methods examined. It can also be seen that GAPSO, PSOGA algorithms have the best MANE values of 0.353 and 0.366 as well as the best RMSE values of 9.080 and 9.466 , respectively.

Table 9 presents the optimized parameters obtained using GA, hybrid GA, PSO, hybrid PSO, and default parameters. For example, the value of "DecelRedDistOwn" and "AccDecelOwn" which is calibrated by GAPSO is $137 \mathrm{~m}$ and $-1.62 \mathrm{~m} / \mathrm{s}^{2}$, in comparison to default values of $200 \mathrm{~m}$ and $-1.00 \mathrm{~m} / \mathrm{s}^{2}$, respectively.

Figure 4 shows the best MANE values obtained by GA, hybrid GA, PSO, and hybrid PSO algorithms with respect to the Number of Function Evaluations (NFE). The value of objective function MANE was calculated by using Equation 1. The x-axis denotes the number of function evaluations and $y$-axis represents the minimum objective function (MANE) value up to every NFE.

Figure 5 shows the best RMSE values obtained by GA, hybrid GA, PSO, and hybrid PSO algorithms with respect to NFE. The value of objective function RMSE was calculated using Equation 2. The x-axis denotes the number of function evaluations and $y$-axis represents the minimum objective function (RMSE) value up to every NFE.

Table 8 - Summary of different objective function values for the optimization problem

\begin{tabular}{|c|c|c|c|c|c|}
\hline \hline Method & Default & GA & PSO & GAPSO & PSOGA \\
\hline \hline MANE & 1.280 & 0.436 & 0.433 & 0.353 & 0.366 \\
\hline RMSE & 34.508 & 11.611 & 11.721 & 9.080 & 9.466 \\
\hline \hline
\end{tabular}

Table 9 - Selected driving behaviour parameter values before and after calibration

\begin{tabular}{||l|c|c|c|c|c|c||}
\hline \multicolumn{1}{|c|}{ Parameters } & Range & Default & GA & PSO & GAPSO & PSOGA \\
\hline \hline W74ax & $0.50 \sim 2.50$ & 2.00 & 1.03 & 0.98 & 1.83 & 1.25 \\
\hline W74bxAdd & $0.70 \sim 4.70$ & 2.00 & 2.88 & 2.42 & 3.18 & 3.03 \\
\hline W74bxMult & $1.00 \sim 8.00$ & 3.00 & 4.55 & 5.89 & 3.90 & 4.52 \\
\hline LookBackDistMax & $50 \sim 200$ & 150 & 112 & 128 & 135 & 127 \\
\hline LookAheadDistMax & $100 \sim 300$ & 250 & 262 & 191 & 195 & 170 \\
\hline StandDist & $0.00 \sim 3.00$ & 0.50 & 1.50 & 1.93 & 0.76 & 1.08 \\
\hline ObsrvdVehs & $1.00 \sim 5.00$ & 2.00 & 2.88 & 3.03 & 2.75 & 3.40 \\
\hline DecelRedDistOwn & $100 \sim 200$ & 200 & 175 & 156 & 137 & 152 \\
\hline AccDecelOwn & $-3.00 \sim 0.50$ & -1.00 & -1.60 & -2.03 & -1.62 & -2.27 \\
\hline MinHdwy & $0.50 \sim 3.50$ & 0.50 & 2.37 & 2.47 & 2.01 & 1.92 \\
\hline SafDistFactLnChg & $0.10 \sim 0.60$ & 0.60 & 0.32 & 0.38 & 0.40 & 0.33 \\
\hline
\end{tabular}




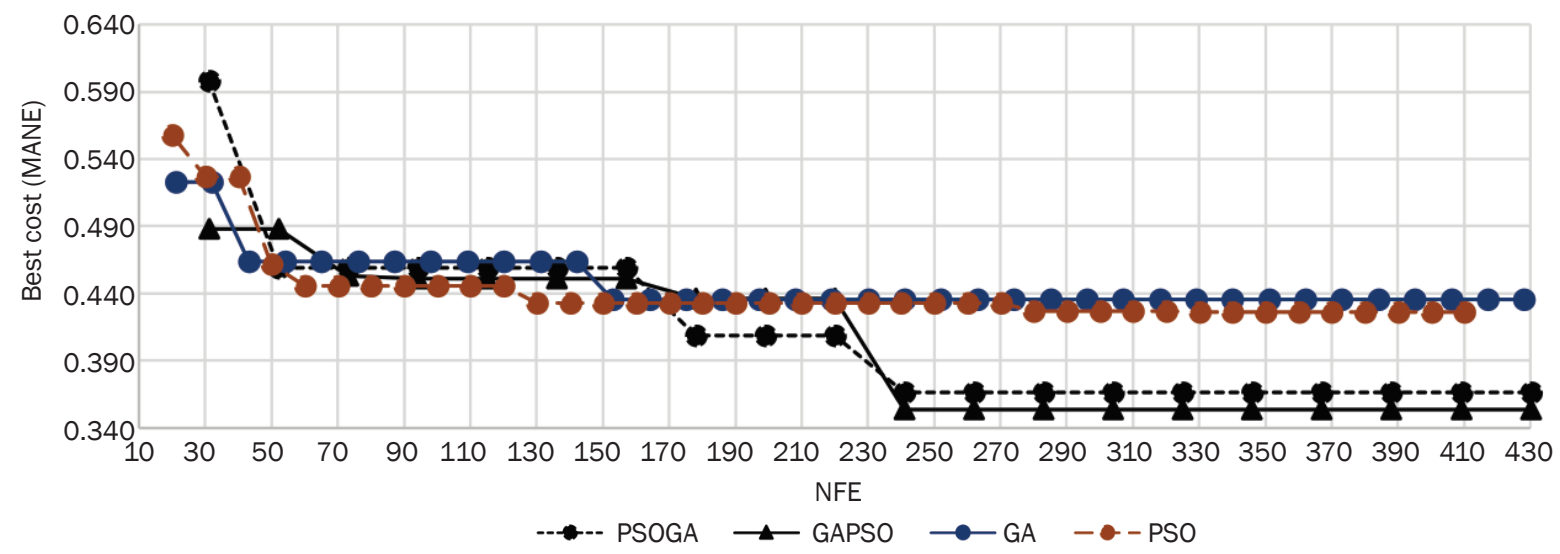

Figure 4 - The best MANE values obtained by hybrid and single optimization algorithms

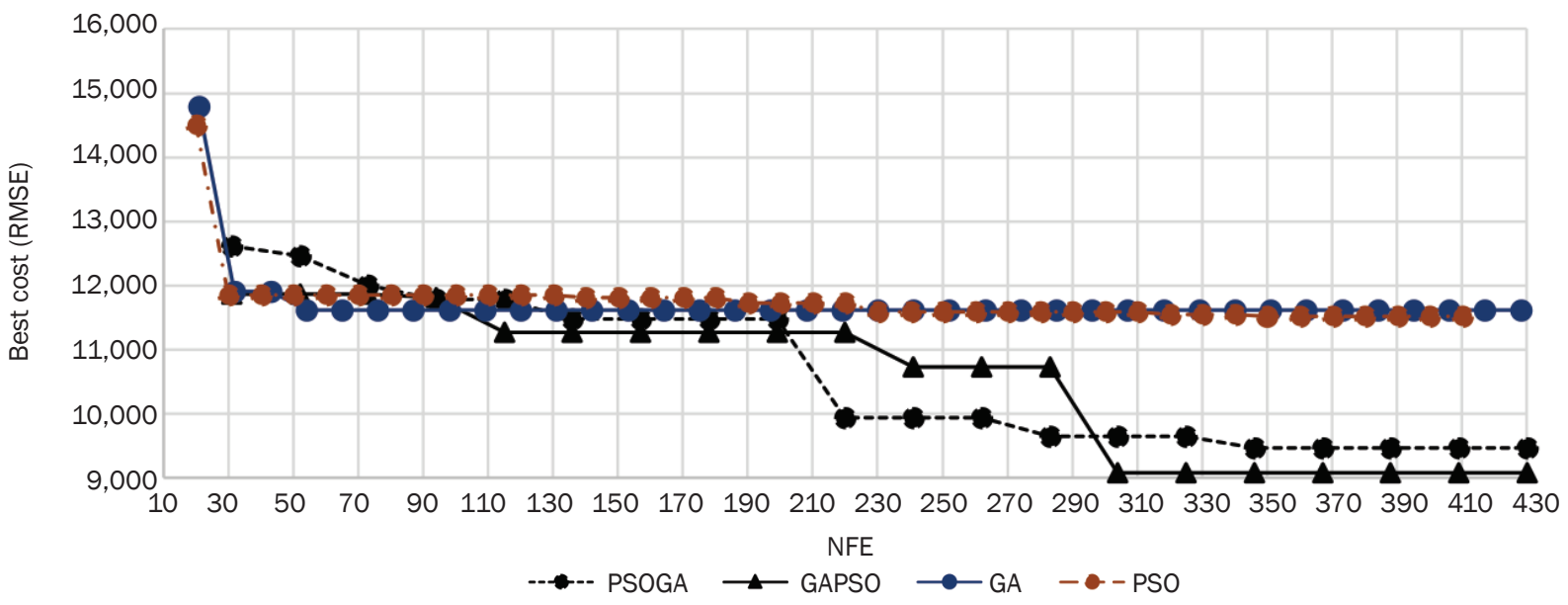

Figure 5 - The best RMSE values obtained by hybrid and single optimization algorithms

One can clearly see that the hybrid algorithms outperform the single algorithms and that the lowest values of both MANE and RMSE are achieved with the hybrid GA algorithm. It is possible to compare the performance of the four algorithms with respect to the percent change from the initial MANE and RMSE scores (1.28 and 34.50) calculated using default values for the selected parameters. Initially, PSO and PSOGA start with the higher MANE values - just above 0.55 , whereas GAPSO registers a better value. After around 240 NFEs, good improvement of MANE values at PSOGA and PSO algorithms could be noticed. Finally, at the end of optimization iterations, hybrid and single algorithms manage to reduce the MANE and RMSE values by $72 \%$, and $66 \%$, respectively, when compared with the initial values.

Figure 6 presents speed profile over selected time period including uncongested flow condition (14:3015:00), transition condition (15:00-15:00), and congested flow condition (>15:20). As shown, the simulated data with calibrated parameters value are in an acceptable fit status, while simulated data with default

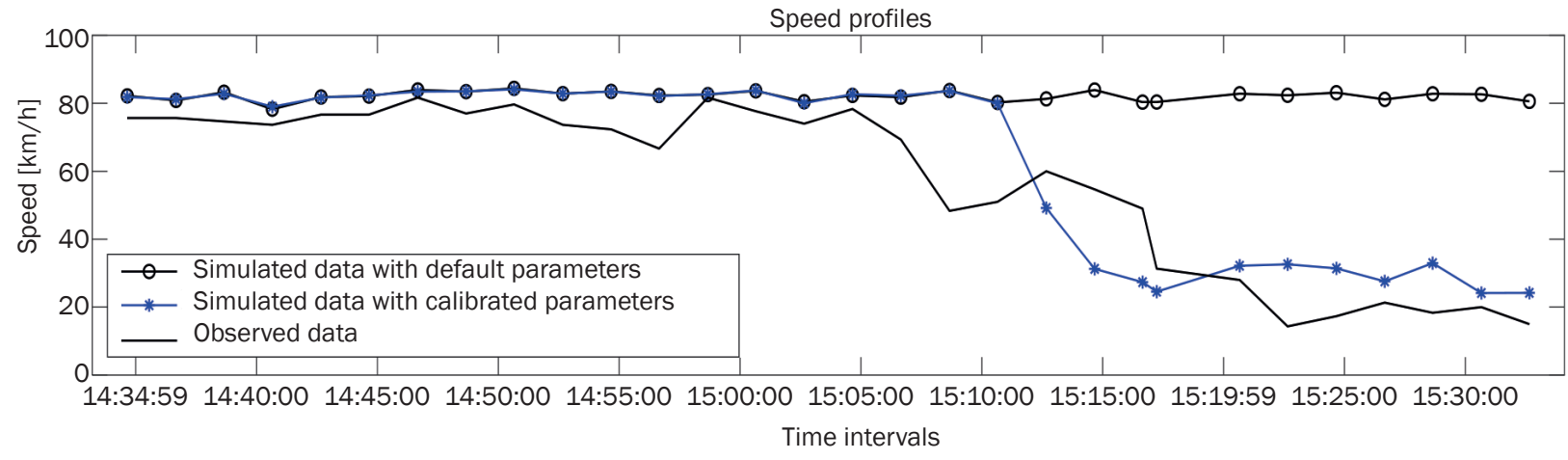

Figure 6 - Speed profiles using default and calibrated parameter values 
parameters value has big difference with the observed data, in particular in transition and congested traffic conditions. As conclusion for our case study, in uncongested flow condition, the simulated models with VISSIM default parameters outputs acceptable results and can be reliable; however, calibrated parameters provide better and well-fit results with the observed data for transition and congested flow condition.

Modelling and calibration processes were done by a personal laptop with the following configuration; CPU: Intel Core ${ }^{\mathrm{TM}}$ i5 - 8500@3.00 GHz, RAM: 16 GB, Operation system: Microsoft windows ver. 10 64-bit. This computer needed around 44 hours to complete optimization for each of the methods.

\section{CONCLUSION}

The interaction between each element creates a great complexity in microsimulation traffic models. The driving behaviour and lane-change model parameters have major effect on the level of representativeness. In order to achieve reliable microsimulation models, more efficient calibration optimization methods should be developed and compared. In this study, metaheuristic optimization methods, namely GA, hybrid GA, PSO, and hybrid PSO, have been developed and applied to calibrate microscopic traffic simulation model parameters. The MATLAB and VISSIM microsimulation software are used for the implementation of the proposed optimization methods. The calibration methods have been implemented and tested in a case study by using traffic data collected from a segment of the 0-1 Highway in Istanbul, Turkey. The observed traffic parameters have been compared with the results obtained by the simulation runs. The calibration is formulated as a minimization problem in which the objective function values are set to MANE and RMSE. The calibration results of objective functions have been presented in detail. Results show that, hybrid GA, and hybrid PSO methods outperform GA-only and PSO-only methods. Among all the algorithms tested, hybrid GA generated the lowest MANE and RMSE values. Based on the achieved results, the combining metaheuristic algorithms approach is very promising and is therefore highly recommended for calibrating microscopic traffic simulation models.

In terms of the future studies, the application of this methodology could be extended to larger freeway networks or signalized roadways. Improving the optimization and calibration performance of the proposed methodology by developing an auto-tuning process for hybrid GA, hybrid PSO, PSO, and GA parameters including "mutation and crossover rates, phi, w, c1, c2, etc." and also by using different combinations of GA and PSO operators inside the hybrid technique remains an interesting area for investigation. Although the proposed calibration methodology shows successful computational performance, one might consider using parallel computing techniques to decrease the calibration time of the proposed calibration procedure [37].

\section{ACKNOWLEDGMENT}

This study is a part of Ph.D. thesis of the corresponding author from the Istanbul Technical University, Turkey. We would like to thank Prof. Dr. Tomaž Maher and Assist. Dr. Rok Marsetič from the Traffic Technical Institute of Civil and Geodetic Engineering Faculty, University of Ljubljana, Slovenia, for their kind support, valuable comments, and helpful suggestions. The authors are also thankful to the PTV-AG Karlsruhe Company for their support in providing a thesis-based unlimited version of VISSIM software.

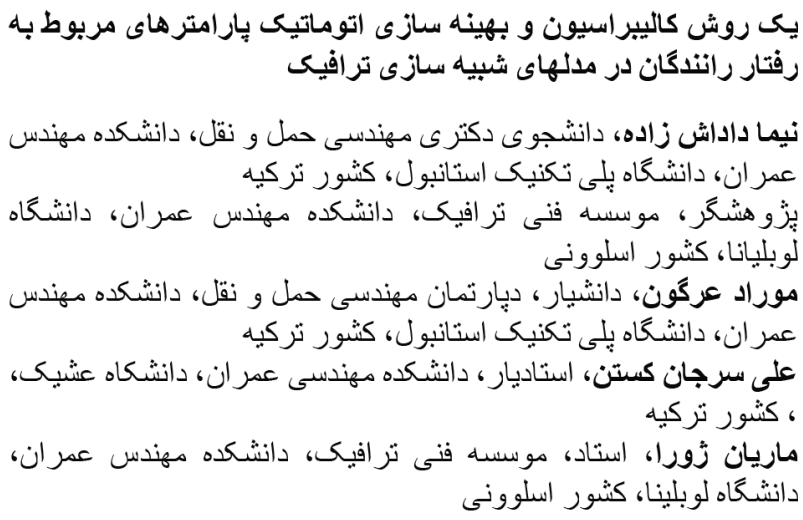

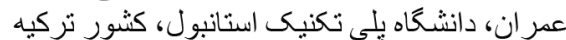

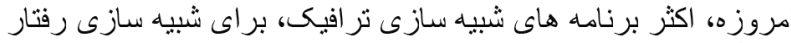

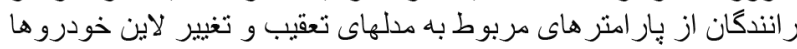

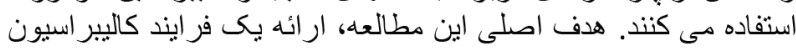

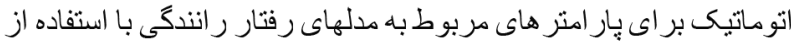

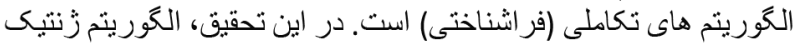

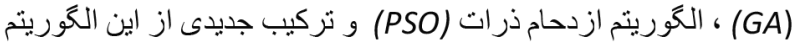
ها يعنى GAPSO و GSOGA در مرحله بينيه سازى استفاده شده است. همجنين دو تابع هدف شناخته شده بعنى MANE , RMSE جها ون كمينه

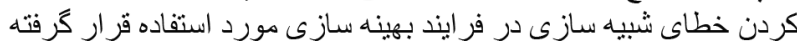

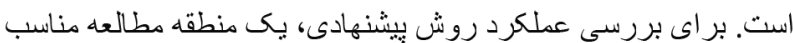

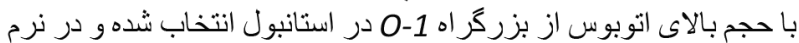

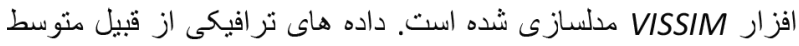

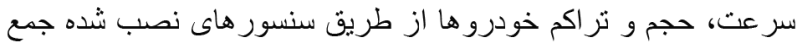

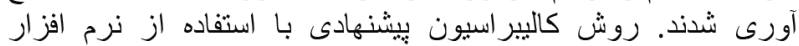

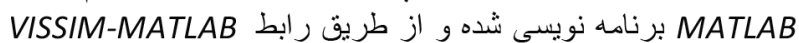

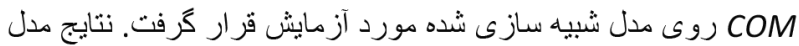

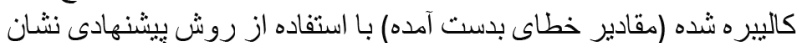

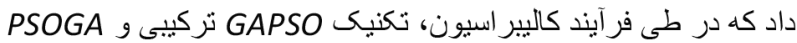
تركيبى نسبت به نكنيك هاى GA تنها و داشتند. بنابر اين، روش بيشنهادى GAPSO تركي كيبى و

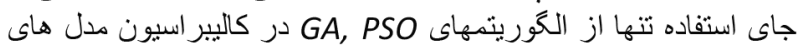
تز افيك توصيه مى شوند.

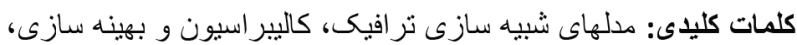

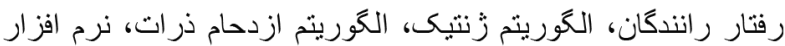
VISSIM 


\section{REFERENCES}

[1] Barcel J, Codina E, Casas J, Ferrer JL, Garcia D. Microscopic traffic simulation: A tool for the design, analysis and evaluation of intelligent transport systems. Journal of Intelligent and Robotic Systems. 2005;41(2-3): 173203. Available from: doi:10.1007/s10846-005-3808-2

[2] Barcelo J, Fellendorf M, Vortisch P. Fundamentals of Traffic Simulation. Springer; 2010. Available from: doi:10.1007/978-1-4614-1900-6

[3] Dowling R, Skabardonis A, Alexiadis V. Traffic Analysis Toolbox Volume III : Guidelines for Applying Traffic Microsimulation Modeling Software. U.S. DOT, Federal Highway Administration, Washington, D.C. Rep. No. FHWA-HRT-04-040, July 2004; 135 p.

[4] Ciuffo B, Punzo V, Torrieri V. Comparison of Simulation-Based and Model-Based Calibrations of Traffic-Flow Microsimulation Models. Transportation Research Record: Journal of the Transportation Research Board. 2008;2088: 36-44. Available from: doi:10.3141/2088-05

[5] Ma J, Dong H, Zhang H. Calibration of Microsimulation with Heuristic Optimization Methods. Transportation Research Record: Journal of the Transportation Research Board. 2007;1999: 208-217. Available from: doi:10.3141/1999-22

[6] Chiappone S, Giuffrè O, Granà A, Mauro R, Sferlazza A. Traffic simulation models calibration using speed-density relationship: An automated procedure based on genetic algorithm. Expert Systems with Applications. 2016;44: 147-155. Available from: doi:10.1016/ j.eswa.2015.09.024

[7] Menneni S, Sun C, Vortisch P. Microsimulation Calibration Using Speed-Flow Relationships. Transportation Research Record: Journal of the Transportation Research Board. 2008;2088: 1-9. Available from: doi:10.3141/2088-01

[8] Strnad I, Žura M. Genetic algorithms application to EVA mode choice model parameters estimation. International Journal of Mathematical Models and Methods in Applied Sciences. 2011;5(3): 533-541.

[9] Hale DK, Antoniou C, Brackstone M, Michalaka D, Moreno AT, Parikh K. Optimization-based assisted calibration of traffic simulation models. Transportation Research Part C: Emerging Technologies. 2015;55: 100115. Available from: doi:10.1016/j.trc.2015.01.018

[10] Boittin C, Gaud N, Hilaire V, Meignan D. Particle swarm for calibration of land-use and transport integrated models. CUPUM 2015 - $14^{\text {th }}$ International Conference on Computers in Urban Planning and Urban Management, 7-10 July 2015, Cambridge, Massachusetts, USA; 2015.

[11] Yu M, (David) Fan W. Calibration of microscopic traffic simulation models using metaheuristic algorithms. International Journal of Transportation Science and Technology. 2017;6(1): 63-77. Available from: doi:10.1016/j.ijtst.2017.05.001

[12] PTV. PTV VISSIM 10 User Manual. PTV Planug Trasport Verker AG, Germany; 2017. p. 265-297.

[13] Zhang Q, Ogren RM, Kong SC. A comparative study of biodiesel engine performance optimization using enhanced hybrid PSO-GA and basic GA. Applied Energy. 2016;165: 676-684. Available from: doi:10.1016/j.apenergy.2015.12.044
[14] Garg H. A hybrid PSO-GA algorithm for constrained optimization problems. Applied Mathematics and Computation. 2016;274: 292-305. Available from: doi:10.1016/j.amc.2015.11.001

[15] Nik AA, Nejad FM, Zakeri H. Hybrid PSO and GA approach for optimizing surveyed asphalt pavement inspection units in massive network. Automation in Construction. 2016;71(Part 2): 325-345. Available from: doi:10.1016/j.autcon.2016.08.004

[16] Katiyar S. A Comparative Study of Genetic Algorithm and the Particle Swarm Optimization. International Journal of Advanced Scientific and Technical Research. 2013;2(3): 98-103.

[17] Kesten AS, Ergün M, Yai T. An Analysis on Efficiency and Equity of Fixed-Time Ramp Metering. Journal of Transportation Technologies. 2013;03(May): 48-56. Available from: doi:10.4236/jtts.2013.32A006

[18] Schaffer JD, Grefenstette JJ. Multi-Objective learning via genetic algorithms. Journal of Chemical Information and Modeling. 1989;53: 160. Available from: doi:10.1017/CB09781107415324.004

[19] Haupt RL, Haupt SE. Practical genetic algorithms. Studies in Computational Intelligence. $2^{\text {nd }}$ Ed. Hoboken, New Jersey: John Wiley \& Sons, Inc.; 2006. p. 7-22. Available from: doi:10.1007/11543138_2

[20] Kennedy J, Eberhart R. Particle swarm optimization. Proceedings of ICNN'95 - International Conference on Neural Networks, 27 Nov - 1 Dec 1995, Perth, WA, Australia. Vol. 4. IEEE; 1995. p. 1942-1948. Available from: doi:10.1109/ICNN.1995.488968

[21] Mezura-Montes E, Coello Coello CA. Constraint-handling in nature-inspired numerical optimization: Past, present and future. Swarm and Evolutionary Computation. 2011;1(4): 173-194. Available from: doi:10.1016/j.swevo.2011.10.001

[22] Lu Z, Fu T, Fu L, Shiravi S, Jiang C. A video-based approach to calibrating car-following parameters in VISSIM for urban traffic. International Journal of Transportation Science and Technology. 2016;5(1): 1-9. Available from: doi:10.1016/j.ijtst.2016.06.001

[23] Ossen S, Hoogendoorn SP. Heterogeneity in car-following behavior: Theory and empirics. Transportation Research Part C: Emerging Technologies. 2011;19(2): 182-195. Available from: doi:10.1016/j. trc.2010.05.006

[24] Abbas MM, Chong L. Car-Following Trajectory Modeling with Machine Learning - A Showcase for the Merits of Artificial Intelligence. 92 ${ }^{\text {nd }}$ Annual Meeting of the Transportation Research Board, 13-17 Jan 2013, Washington DC, USA; 2013.

[25] Menneni S, Sun C, Vortisch P. Microsimulation Calibration Using Speed-Flow Relationships. Transportation Research Record: Journal of the Transportation Research Board. 2008;2088: 1-9. Available from: doi:10.3141/2088-01

[26] Durrani U, Lee C, Maoh H. Calibrating the Wiedemann's vehicle-following model using mixed vehicle-pair interactions. Transportation Research Part C: Emerging Technologies. 2016;67: 227-242. Available from: doi:10.1016/j.trc.2016.02.012

[27] Lu X, Lee J, Chen D, Bared J, Dailey D, Shladover SE. Freeway Micro-simulation Calibration: Case Study Using Aimsun and VISSIM with Detailed Field Data. Transportation Research Board 93 ${ }^{\text {rd }}$ Annual Meeting. 12-16 
Jan 2014. Washington, D.C., USA; 2014; p. 1-17.

[28] Hourdakis J, Michalopoulos P, Kottommannil J. Practical Procedure for Calibrating Microscopic Traffic Simulation Models. Transportation Research Record: Journal of the Transportation Research Board. 2003;1852: 130-139. Available from: doi:10.3141/1852-17

[29] Lee J-B, Ozbay K. New Calibration Methodology for Microscopic Traffic Simulation Using Enhanced Simultaneous Perturbation Stochastic Approximation Approach. Transportation Research Record: Journal of the Transportation Research Board. 2009;2124: 233240. Available from: doi:10.3141/2124-23

[30] Hollander Y, Liu R. The principles of calibrating traffic microsimulation models. Transportation. 2008;35(3): 347-362. Available from: doi:10.1007/s11116-0079156-2

[31] Barroso ES, Parente E, Cartaxo de Melo AM. A hybrid PSO-GA algorithm for optimization of laminated composites. Structural and Multidisciplinary Optimization. 2017;55(6): 2111-2130. Available from: doi:10.1007/ s00158-016-1631-y

[32] Liang Z, Ouyang J, Yang F. A hybrid GA-PSO optimization algorithm for conformal antenna array pattern synthesis. Journal of Electromagnetic Waves and Applications. 2018;32(13): 1601-1615. Available from: doi:1 0.1080/09205071.2018.1462257

[33] Sheikhalishahi M, Ebrahimipour V, Shiri H, Zaman H, Jeihoonian M. A hybrid GA-PSO approach for reliability optimization in redundancy allocation problem.
International Journal of Advanced Manufacturing Technology. 2013;68(1-4): 317-338. Available from: doi:10.1007/s00170-013-4730-6

[34] Dadashzadeh N, Ergun M. Spatial bus priority schemes, implementation challenges and needs: an overview and directions for future studies. Public Transport. 2018;10(3): 545-570. Available from: doi:10.1007/ s12469-018-0191-5

[35] Alpkokin P, Black JA, lyinam S, Kesten AS, Alpkokin P, Black JA, et al. Historical analysis of economic , social and environmental impacts of the Europe-Asia crossings in Istanbul. International Journal of Sustainable Transportation. 2016;10(2): 65-75. Available from: do i:10.1080/15568318.2013.853852

[36] Alpkokin P, Ergun M. Istanbul Metrobüs: first intercontinental bus rapid transit. Journal of Transport Geography. 2012;24: 58-66. Available from: doi:10.1016/j. jtrangeo.2012.05.009

[37] Dadashzadeh N, Ergun M, Kesten AS, Žura M. Improving the calibration time of traffic simulation models using parallel computing technique. In: Kucharski $R$, Szarata A (eds.) IEEE MT-ITS2019 $6^{\text {th }}$ International Conference on Models and Technologies for Intelligent Transportation Systems. Kraków, Poland. IEEE Xplore; 2019. p. 54. Available from: https://mt-its2019.syskonf.pl/conf-data/mt-its2019/files/MT-ITS2019 Book of Abstract 4_06.pdf 